\title{
Key Points of Narrow Reading in Medical English-An Empirical Study Based on Stomatological English
}

\author{
Ying Feng1, Lihua $\mathbf{L i}^{2}$ \\ ${ }^{1}$ Department of Foreign Language and Culture, North Sichuan Medical College, Nanchong, China \\ ${ }^{2}$ Department of Stomatology, North Sichuan Medical College, Nanchong, China \\ Email: fengying202112@126.com
}

How to cite this paper: Feng, Y. and Li, L.H. (2021) Key Points of Narrow Reading in Medical English-An Empirical Study Based on Stomatological English. Open Access Library Journal, 8: e8069. https://doi.org/10.4236/oalib.1108069

Received: October 12, 2021

Accepted: December 10, 2021

Published: December 13, 2021

Copyright $\odot 2021$ by author(s) and Open Access Library Inc.

This work is licensed under the Creative Commons Attribution International License (CC BY 4.0).

http://creativecommons.org/licenses/by/4.0/ (c) (i) Open Access

\begin{abstract}
This paper based on the results of an empirical study which concerns the application of narrow reading on Stomatological English, analyzes the selection of reading material, the principle for theme and the implementation requirements of narrow reading and thus sharpens the rational cognition. This paper discusses the effects of narrow reading on improving students' professional knowledge and English. Moreover, this paper provides valuable references of applications of narrow reading in medical English reading.
\end{abstract}

\section{Subject Areas}

Linguistics

\section{Keywords}

Narrow Reading, Medical English, Reading Teaching

\section{1. 引言}

20 世纪 80 年代, 国学者 Stephen Kreshen 以及 Sperber 等先后提出的窄 式阅读理论(Narrow Reading), 国内结合窄式阅读理论对商务英语专业的阅读 情况、窄式阅读概述、对高职英语教学中的有效性研究、应用方式、词汇学 习等方面有系列探讨。窄式阅读[1]在学生专业词汇积累、阅读理解的能力提 高及以读促写等方面产生的积极作用, 在《阅读强化与窄式阅读对提高非英 语专业学习者词汇习得的比较研究》[2]、《窄式阅读在高职英语教学中的应 用》[3]、《浅析窄式阅读在商务英语中的应用》[4]、《窄式阅读对促进高中 英语写作教学的实证研究》[5]等文章中得到了充分的证明与阐释。然而, 综 
合分析中国知网上近五年来(2015 2019)收录的关于窄式阅读的文献发现多 数研究者的着眼点是 “应用”, 且聚焦于应用的结果。本文作为笔者主研的 针对口腔专业学生实施的窄式阅读研究课题(课题编号 SCWYH13-11)的后续 成果, 及编写校内自用教材《口腔医学双语教程(英文版)》的编撰思考, 跳 出之前的常规研究思路, 深入探讨了能决定应用结果的目标确立、选材和实 施三大要素, 形成了对窄式阅读理论的全面认识和深层理解。

\section{2. 目标确立的原则}

在 “双一流” 和 “一带一路” 的建设背景下, 高校外语教育从通用英语 教学转向专门用途英语教学成为趋势。医学英语是专门用途英语的一个分支, 也是医学生和医务人员用英语从事专业学习和工作的极大助力。对不同医学 专业的学生进行窄式阅读训练的目标正是在于提高学生在学科领域内应用英 语的能力。笔者通过主持的窄式阅读实证研究过程, 认为窄式阅读训练应遵 循以下原则, 方能取得良好的效果:

\section{(一) 落实 《大学英语教学指南》的相关要求}

2015 年, 受教育部委托, 高等学校大学外语教学指导委员会在总结大学 英语课程建设和教学改革经验的基础上, 研究制定了《大学英语教学指南》 [6]。《教学指南》成为新时期普通高等学校制订大学英语教学大纲, 进行大 学英语课程建设, 开展大学英语课程评价的依据。其中, 课程定位与性质的 第二条课程性质的阐述中, 明确指出: 大学英语的工具性也体现在专门用途 英语上, 学生可以通过学习与专业或未来工作有关的学术英语或职业英语, 获得在学术或职业领域进行交流的相关能力。《教学指南》中三个级别的英 语教学对阅读技能的要求如下:

基础目标: 能借助词典阅读英语教材和未来工作、生活中常见的应用文 和简单的专业资料。

提高目标: 能阅读与所学专业相关的综述性文献, 理解中心大意、关键 信息、文章的篇章结构和隐含意义等。

发展目标: 能比较顺利地阅读公开发表的英语报刊上的文章, 以及与所 学专业相关的英语文献和资料, 较好地理解其中的逻辑结构和隐含意义等 [6]。

基于此表述不难看出阅读能力也是专门用途英语的教学关注点之一。窄 式阅读训练正是着眼于 “培养学生专业英语的阅读能力”, 以区别于传统的 阅读教学, 来达到促进医学英语课程在发展学生语言运用能力、进而提高学 生的学术探究能力方面的作用。窄式阅读主张进行同一主题的阅读, 这非常 符合不同专业的医学生的实际学习需求。学生在完成主题性阅读的过程中可 能对某一学科问题产生兴趣并进行深入的学习和研究, 从而锻炼和发展独立 探究的能力。在深入研究的时候又需要应用英语去获取新信息, 从而促进学 生的语言运用能力。因此, 窄式阅读训练能落实《教学指南》提出的阅读能 力和应用技能的要求。

\section{(二) 关注学生的现有水平和实际需求}

在进行窄式阅读训练时, 必须关注学生的现有水平, 即学生的学科知识 
水平和英语语言水平。如果阅读材料的语言和涉及的学科内容都比较浅显的 话, 就不能激发起学生深入探究的兴趣; 相反, 阅读材料的语言和涉及学科 内容过于深奥难解, 学生也会因力不能及而望之兴叹甚至放弃。大学生的实 际需求包括了发展过程中的需求和未来的需求。发展过程中的需求是以学生 在提升总体能力的过程中形成某种具体能力为目标, 解读《教学指南》可以 知道专业英语阅读的能力也是目标的一部分; 而学生未来的需求以学生最终 具备在专业领域内熟练应用英语的能力为目标。因此, 窄式阅读训练不能脱 离这两方面的约束。

\section{(三) 鼓励学生自选主题}

学生自主选择主题, 是窄式阅读开放性和适应性的体现。既达到丰富 “主 题” 的效果, 也能激发学生参与的热情和学习的兴趣。对此, 教师可以提供 材料, 可以提出问题, 可以布置任务, 学生根据材料、问题和任务来确定自 己感兴趣并符合自身需要的一些主题; 而在分析材料、解决问题和完成任务 的过程中, 又有可能大主题下发散出新的小主题。这样, 学生自选主题的过 程也是学生提升专业素养、增强能力的过程。学生自选主题时, 教师应当给 予一定的指导。让学生的主题内容更具有针对性, 更接近学习的目标要求, 更适合于学生的实际水平和需要。鼓励学生自选主题, 也是促进师生共同发 展的一种方式。

\section{3. 窄式阅读模式中阅读材料的选择}

信息化时代, 进行选择性的有效阅读尤为重要。在医学英语的阅读教学 中, 材料的选择是不能忽视的一方面。目前, 从已发表的文献资料上看, 教 师对用于窄式阅读训练的阅读材料的选择基本上达到了克拉申在窄式阅读理 论中明确提出的两个基本要求, 即 “可理解性输入” (材料的语言水平)和 “同 一主题” (材料的学科内涵)。但笔者通过亲历的实证研究发现除上述两点外, 阅读材料, 尤其在材料的学科内涵方面还应该满足下列条件才能更好地体现 窄式阅读的优点。

\section{(一) 材料的延伸性}

大学阶段的学生处于集中获取专业知识的关键时期, 其知识体系的发展 开始出现梯形结构的特征。因此, 窄式阅读材料在学科内涵上应具备延伸性。 延伸性不只是简单的内容上的扩展, 而是以内容扩展为手段, 帮助学生形成 关于学科内容的更深刻认知, 即培养学生关于某学科的学术思维能力和促进 学生自身学术观点的初步形成。在此可以借鉴杰出的教育心理学家维果茨基 的 “最近发展区” 理论来安排和设计用于窄式阅读的阅读材料。

第一, 所选材料的学科内容范围应该呈梯形结构。第二, 材料的内容难 度安排上应适合学生的专业知识水平发展, 并略高于现有水平(类似于材料在 语言水平层面满足 “可理解性输入” 的要求)。这样, 学生在窄式阅读中接触 到的内容与其知识体系发展的特征相匹配; 那么在教师的帮助下学生就能顺 利地达到更高一点的知识认知水平。因此, 学习过程中学生可以逐步就某学 科域甚至就某个专门的问题作更深入的学习, 这一过程对学生的独立探究能 力或学术能力的发展大有裨益。 
现以针对口腔专业开设的窄式阅读研究为例说明。口腔专业开设的专业 课程有 14 门左右, 如: 口腔解剖生理学、口腔组织病理学、口腔修复学等。 在窄式阅读训练过程中, 教师按口腔解剖生理学和口腔组织病理学(基础内 容), 口腔内科学、口腔领面外科学和口腔修复学(进阶内容), 口腔正畸学(高 级内容)这样一个梯形的方式来安排学生的阅读材料。这样安排有效地配合了 学生专业知识的学习顺序并体现了知识的连续和延伸性, 避免了阅读材料虽 满足语言层面的条件, 但阅读内容前后有脱节的情况。实验后的随访反映学 生觉得按此顺序进行的阅读训练本身就是一个完整的体系, 而不是零散的材 料拼凑。受试学生中认可这一安排的比例达 $96.7 \%$ (62 名受试, 60 名认可)。

\section{(二) 材料的多样性}

窄式阅读的 “窄” 是指材料主题的接近性, 但并不排斥材料本身的多样 性。这意味着教师可在主题接近的大前提下尽可能地为学生提供充足的阅读 内容。在同一主题下, 教师可为学生提供不同体裁的材料, 材料的内容也可 细化到该主题的各方面。还以针对口腔专业开设的窄式阅读研究为例。把作 为进阶内容的口腔内科学的相关阅读材料的重点集中在龀病这一大主题上。 除了提供给学生关于该病种的病因、临床表现和常规治疗的阅读材料外, 再 提供关于该病的新疗法等这样一些小主题的阅读材料, 这样做既可以提起学 生的阅读兴趣, 也可以深化学生的知识水平。为满足学生选择的多样性, 还 可以提供关于齫病的科普性文章、临床病例分析以及学术文献等不同体裁的 阅读材料。

\section{(三) 材料的灵活性}

如果说前面提到的 “延伸性” 和 “多样性” 主要是教师依据教学目的来 选材料的话, 那么材料的灵活性则从学生的兴趣爱好、需求程度出发, 体现 了窄式阅读对学生的兴趣和需要变化的适应性。教师可以发动学生根据自己 的接受能力和实际需要去选择阅读主题, 借助网络、报刊杂志等媒体进行材 料选择。笔者认为这些材料可以篇幅短小, 用语简练但有学科特征(如 VOA 健康报道的文字材料), 适用于制作活页读本, 作为有组织的窄式阅读的补充, 既方便学生随时增减内容, 也方便学生之间交换阅读。

使用满足上述条件的阅读材料时, 学生需要完成从理解语言到理解内容, 从理解内容中的一点到理解内容的体系这样一个过程。因此, 每一个主题的 完成都覆盖了语言和知识两方面的学习。这样, 通过多条途径来实现医学英 语阅读教学应达到的目标。

\section{4. 窄式阅读训练的实施要求}

\section{（一）对于每个主题要有总体的设计}

首先需要澄清的一个认识误区是进行窄式阅读训练所选主题越多越好。 主题多少的选择度应该以学科重点为参考。训练也应保证选择的每一个主题 都能够为实现医学英语教学的总体要求服务。因此, 对每个主题进行总体的 设计是非常必要的。例如, 在对列为高级内容的口腔正畸学部分进行窄式阅 读训练时, 将 “常见错牙合畸形及矫治” 作为一个大主题。围绕这个主题, 精选关于错牙合畸形的分类, 临床诊断和治疗的英文原版教材作为阅读材料, 
了解文章所传达的思想观点。在此基础上, 设计 “安氏 I 类错牙合的诊疗” “安氏 II 类错牙合的诊疗” 和 “安氏 III 类错牙合的诊疗” 三个单元主题并 分别细化到如 “安氏 I 类错牙合的诊段方法”、“安氏 I 类错牙合的矫治措 施” 等六个重点方面。进行总体设计便于师生双方把握阅读训练的进程及检 验阅读效果。进行总体设计时应考虑实现 “提升学生专业素养和发展语言能 力” 这一核心目标。其实施过程既是对教材的消化吸收甚至深挖掘, 也是学 生语言能力的提升。

\section{(二) 组织窄式阅读训练要有合理明晰的流程}

传统医学英语课堂, 大多是一次课就是一个相对完整的过程, 形成一个 “板块”。而窄式阅读训练则打破了这种惯例。窄式阅读训练一般是以一个 主题形成一个 “板块” 。如该主题需用几节课来完成, 那么建立一个合理的 教学流程是必要的。例如, 就 “安氏 II 类错牙合的治疗” 这一主题先安排学 生阅读教师提供的相关资料, 进行分析, 然后与同学交流, 最后完成制定病 例矫治方案的作业并展示。从本次研究过程可以看出从 “材料研究” 到 “相 互交流” 再到 “成果展示” 是一个基本的过程。

\section{（三）根据实际情况对选择的主题作出调整}

是否进行调整主要取决于下面几个因素。首先是学生的学习兴趣。学生 会因多种原因而不能在研究的过程中始终对主题保持兴趣。此时就需要对已 选定的主题作一点调整, 尽可能地保持学生的兴趣。其次是不同学生的接受 程度。同一个主题, 学生的接受度因人而异, 教师可以在难度、范围、目标 等方面做出一些调整。最后是课时的进度。有的主题, 学生做起来可能感觉 很有意思, 那就可以适当延长一点时间。后面受到影响的专题就相应作出调 整。同样, 有的主题, 学生所用时间比预期要少一些, 也没必要非按照原计 划执行。简言之, 窄式阅读训练在实施过程中也存在实际与预设有出入的问 题, 那么, 进行内容、数量、时间和方法上的调整都是可以的。关注学生, 随 “学情” 灵活掌握。

\section{5. 结语}

窄式阅读训练通过满足学生的不同兴趣和需要最终要达到的目的是服务 于《教学指南》对专门用途英语的总体要求, 最终要收获的是学生在专业素 养和英语应用能力两方面的提升。

\section{Conflicts of Interest}

The authors declare no conflicts of interest.

\section{References}

[1] Krashen, S. (2004) The Case for Narrow Reading. Language Magazine, 3, 17-19. http://www.sdkrashen.com/content/articles/narrow.pdf

[2] 王永基. 阅读强化与窄式阅读对提高非英语专业学习者词汇习得的比较研究 [D]: [硕士学位论文]. 兰州: 西北师范大学, 2016.

[3] 张旭丹. 窄式阅读在高职英语教学中的应用[J]. 课程教育研究, 2018(52): 60. 
[4] 禹瑶, 徐婧玉, 武则地. 浅析窄式阅读在商务英语中的应用 [J]. 现代交际, 2019(2): 16-17.

[5] 李多. 窄式阅读对促进高中英语写作教学的实证研究[D]: [硕士学位论文]. 哈尔 滨: 哈尔滨师范大学, 2019.

[6] 360 文库. 大学英语教学指南(教育部最新版) [R/OL].

https://wenku.so.com/d/43089cafb1eb8dcefa218da7203a0d69, 2020-03-28.

\section{Appendix (Abstract and Keywords in Chinese)}

\section{窄式阅读在医学英语阅读教学中的实施要领——基于口腔医学英语实 证研究的理论阐述}

摘要: 本文基于窄式阅读运用于口腔医学英语阅读教学的实证研究结果, 深 入思考了窄式阅读过程中目标确立的原则、阅读材料的选择及窄式阅读的实 施要求等三要素, 从而深化对窄式阅读的理性认知。本文以期为窄式阅读在 医学英语的教学实践及相关学术研究方面提供新思路及参考。

关键词: 窄式阅读, 医学英语, 阅读教学 